Supporting Information

\title{
Red Algae-Derived Carrageenan Coatings for Marine Antifouling Applications
}

\author{
Dahee Kim and Sung Min Kang* \\ Department of Chemistry, Chungbuk National University, Chungbuk 28644, Republic of \\ Korea \\ * Corresponding Author \\ E-mail: smk16@chungbuk.ac.kr
}

Number of pages: 4

Number of table: 1

Number of figures: 4 
Table S1. Previous and current approaches to fabricate marine antifouling polysaccharide coatings.

\begin{tabular}{ccccc}
\hline & Polysaccharide & $\begin{array}{c}\text { Solid } \\
\text { substrate }\end{array}$ & Coating layers & $\begin{array}{c}\text { External } \\
\text { equipment }\end{array}$ \\
\hline $\begin{array}{c}\text { Previous } \\
\text { approach }\end{array}$ & $\begin{array}{c}\text { Limited to carboxyl- } \\
\text { containing polysaccharides }\end{array}$ & $\begin{array}{c}\text { Specific } \\
\text { substrates }\end{array}$ & Single layer coating & Not required \\
\hline $\begin{array}{c}\text { Current } \\
\text { approach }\end{array}$ & $\begin{array}{c}\text { Not limited to carboxyl- } \\
\text { containing polysaccharides }\end{array}$ & $\begin{array}{c}\text { Various } \\
\text { substrates }\end{array}$ & $\begin{array}{c}\text { Thickness- } \\
\text { controllable } \\
\text { multilayer coating }\end{array}$ & Required \\
\hline
\end{tabular}

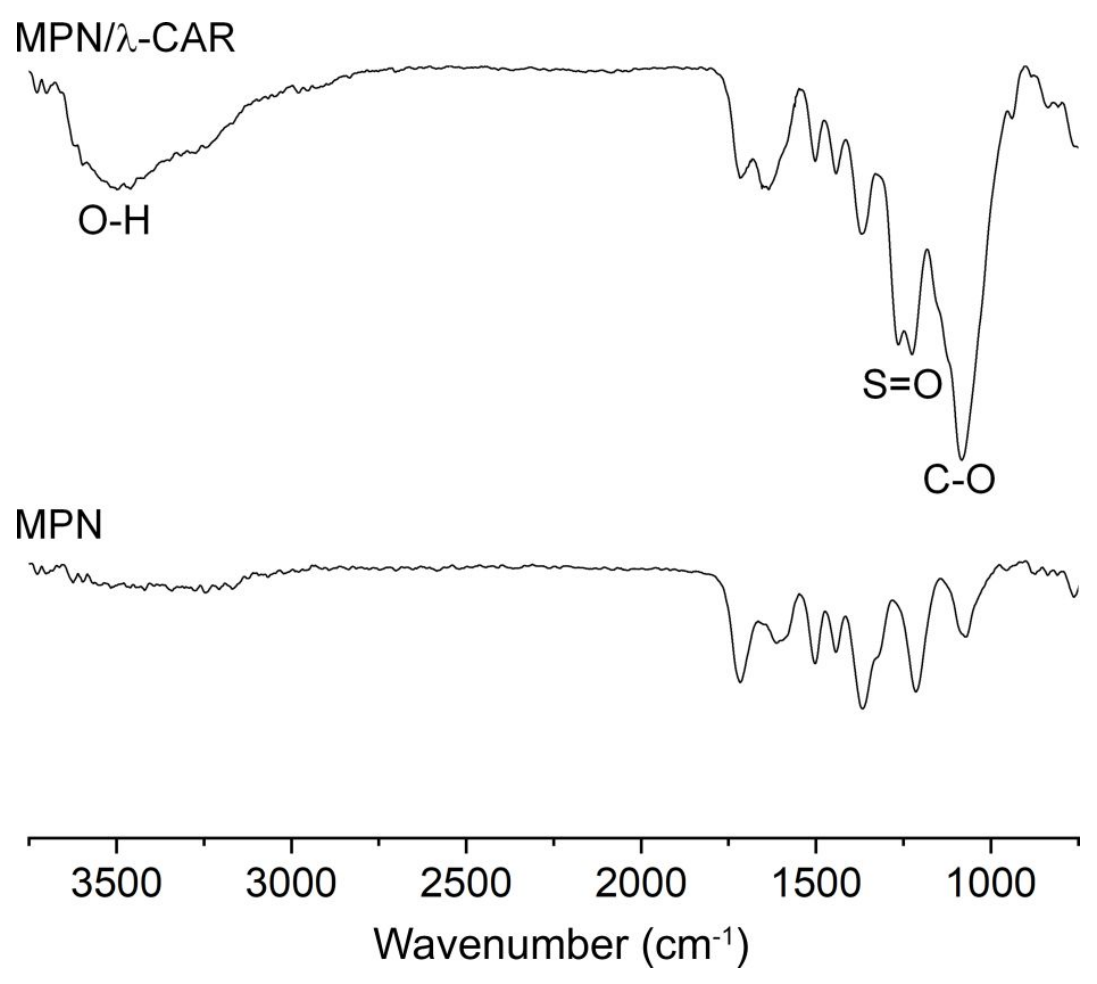

Figure S1. FT-IR spectra of MPN-coated and MPN/ $\lambda$-CAR-coated Ti/TiO 2 surfaces. MPN, metalpolyphenol network; CAR, carrageenan. 

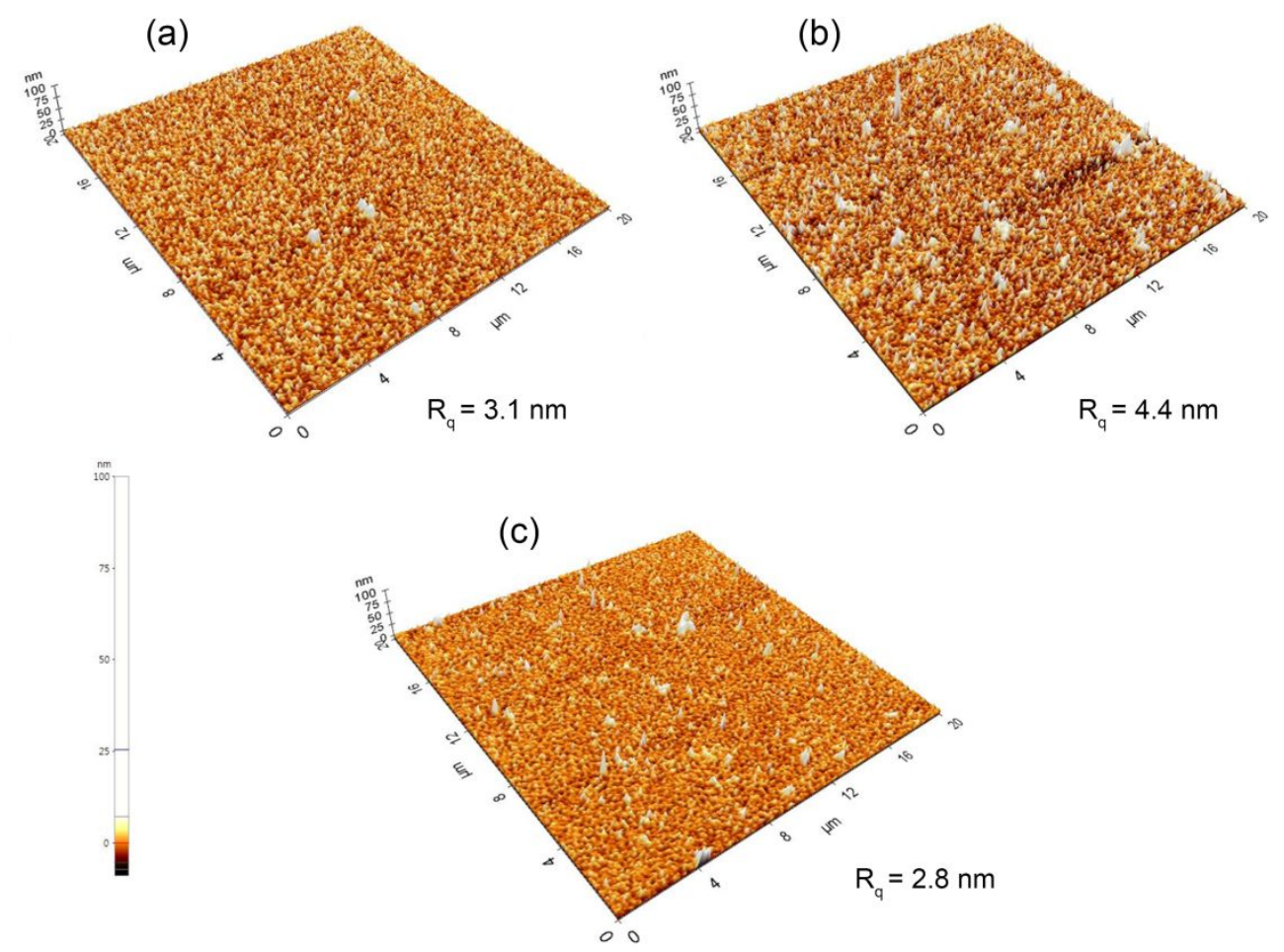

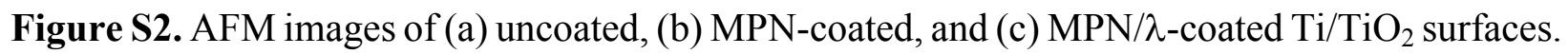
MPN, metal-polyphenol network; CAR, carrageenan.

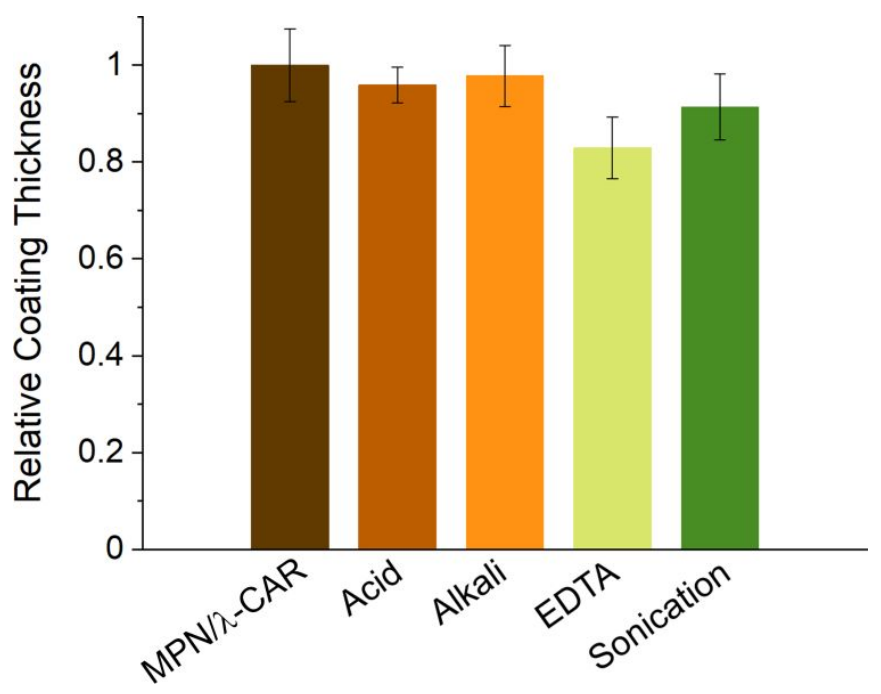

Figure S3. Relative thickness change of MPN/ $\lambda$-CAR coatings after acid (10 mM HCl), alkali (10 $\mathrm{mM} \mathrm{NaOH}$, EDTA, and sonication treatments. MPN, metal-polyphenol network; CAR, carrageenan. 
MPN-coated Nylon

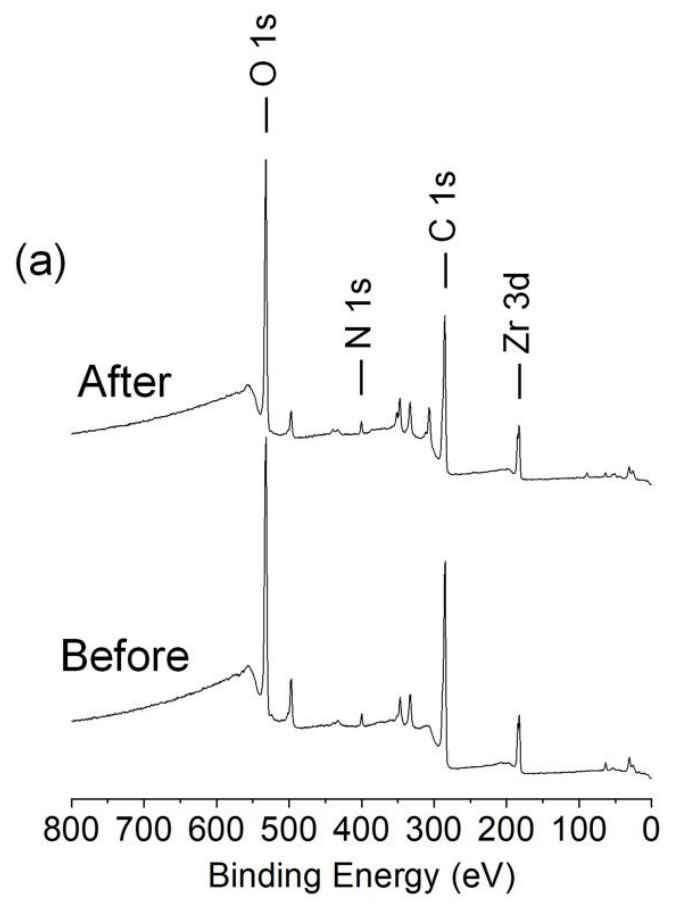

(b)

\begin{tabular}{ccccc}
\hline$\%$ & C 1s & N 1s & O 1s & Zr 3d \\
\hline Before & 62.65 & 1.32 & 33.51 & 2.52 \\
After & 59.84 & 1.53 & 35.83 & 2.80 \\
\hline
\end{tabular}

MPN-coated Glass

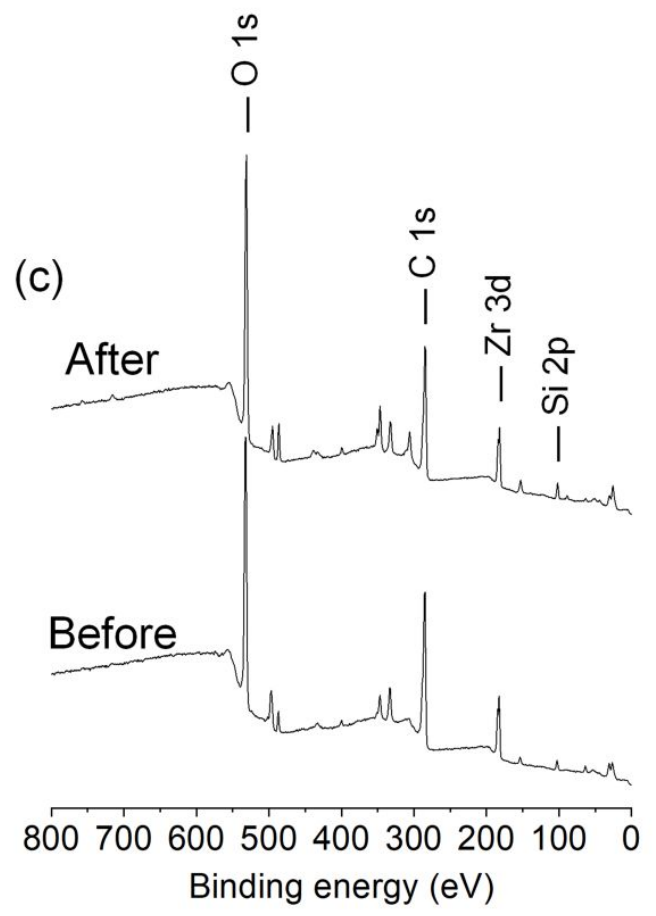

(d)

\begin{tabular}{ccccc}
\hline$\%$ & C 1s & Si 2p & O 1s & Zr 3d \\
\hline Before & 57.89 & 2.55 & 36.76 & 2.79 \\
After & 54.59 & 4.38 & 38.16 & 2.87 \\
\hline
\end{tabular}

Figure S4. XPS spectra (a and c) and atomic composition (\%) (b and d) of MPN-coated Nylon and MPN-coated Glass surfaces before and after immersion in seawater for one week. 